\title{
Desafios na construção de um ambiente adaptativo baseado em ncias Múltiplas com apoio de gamificação
}

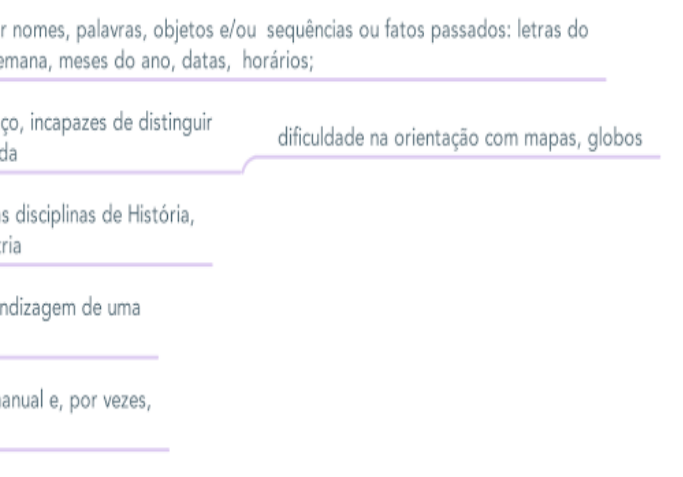

dades com a matemática, Sobretudo na assimilação de símbolos e em decorar a tabuada.

\author{
n X. Pereira Júnior ${ }^{1}$, Márcia A. Fernandes ${ }^{2}$ \\ ${ }^{1}$ Instituto Federal Goiano (IF Goiano) \\ Campos Belos - GO - Brasil \\ ¿omputação - Universidade Federal de Uberlândia (UFU) \\ Uberlândia - MG - Brasil \\ -junioreifgoiano.edu.br, marcia@ufu.br
}

he Challenges of Informatics in Education is the adaptation ning environments and automatic modeling of the student. 'ows a proposal of a learning environment based on Multiple xpt contents and form the student model. A module of gamifissary to motivate the student to help in the choice of the best st effectuation of the learning. We believe that the creation t can facilitate the proccess of distance education, helping in on and low yield.

desafios apresentados pela Informática na Educação é a teúdos em ambientes de aprendizagem e a modelagem auinte. Desta forma, este trabalho apresenta uma proposta de um ambiente baseado em Inteligências Múltiplas para a adaptação do conteúdo e formação do modelo do estudante. Um módulo de gamificação se faz necessário para motivar o aprendiz e o auxiliar na escolha do melhor cenário para a melhor efetivação da aprendizagem. Acredita-se que a criação de tal ambiente possa facilitar o processo de Educação a Distância, auxiliando na diminuição da evasão e do baixo rendimento.

\section{Introdução}

A tecnologia, atualmente, encontra-se presente e desafiadora em todas as modalidades de ensino. Nas modalidades à distância, ou semipresenciais, os desafios norteiam, principalmente, na escolha da ferramenta para a melhor abordagem do conteúdo didático. Pesquisadores da área de Informática na Educação (IE) vêm sendo instigados a propor sistemas educacionais adaptativos, isto é, aqueles que melhor se adaptem ao modelo do estudante, individualizando / personalizando assim o processo ensino / aprendizagem. Atualmente, é esperado que um ambiente virtual auxilie estudantes e professores na disponibilização e organização dos materiais de ensino para uma aprendizagem mais efetiva.

No Brasil, existem alguns programas que incentivam a Educação a distância (EaD). Através da Universidade Aberta do Brasil (UAB) e de outros programas, o governo vem incentivando, na modalidade a distância, a formação técnica e superior dos cidadãos. Tal alternativa vem ampliando nos últimos anos o maior acesso a educação por parte da população brasileira. 
Apesar dos programas apresentarem grandes vantagens, estudos recentes ainda mostram um número considerável de evasões e reprovações nestas modalidades [Martins et al. 2016, Fernandes et al. 2014]. A falta de acesso aos ambientes virtuais e dificuldades na adaptação à metodologia são apresentados como fatores que levam à reprovação e à evasão dos aprendizes [Fernandes et al. 2014]. Desta forma, é possível crer que Sistemas dotados de inteligência ainda podem ser grandes aliados para mudarmos o cenário atual.

Pesquisadores têm proposto com mais frequência sistemas que empregam técnicas de Inteligência Artificial (IA) com o objetivo de identificar características específicas do estudante e, consequentemente, inferir ações pedagógicas apropriadas que melhoram a pratica do ensino. Por exemplo, acredita-se que cada estudante tenha um Estilo de Aprendizagem (EA) e que, aplicadas em ambientes virtuais, podem favorecer a aprendizagem do sujeito [Graf et al. 2010].

Ainda no contexto da aprendizagem, a teoria das Inteligências Múltiplas (IM) pode desempenhar um papel semelhante ao esperado dos EA [Feldman et al. 2015]. Segundo Gardner [Gardner 1983], o ser humano possui inteligências, sendo que algumas delas podem destacar. [Barbosa et al. 2004], em sua pesquisa, conseguiu associar mídias com algumas inteligências, desta forma, é possível analisar situações em que Objetos de Aprendizagem (OA) sejam elaborados para coincidir com a inteligência em destaque, tornando assim a aprendizagem mais efetiva. Assim, um estudante que possui a inteligência lógico-matemática em alto grau, materiais que envolvem associação e raciocínio podem ser apresentados a este estudante tentando assim levar uma maior absorção.

Um desafio ainda existente na pesquisa são métodos para aferição de IM's na intenção de modelar o estudante. O questionário é o método mais tradicional e o principal utilizado para tal medição, embora Gardner [Gardner 1983] critique esta prática. O mesmo desafio ocorre para a aferição dos EAs, também sendo tradicionalmente medidos por questionários. Alguns trabalhos já apresentam uma evolução na estratégia de medição [Graf et al. 2010], porém ainda é um problema em aberto na pesquisa.

Por ser um problema em aberto, estratégias distintas devem ser adotadas visando melhores soluções. Desta maneira, a gamificação pode ser uma tentativa para auxiliar no processo. A gamificação, técnica utilizada nos últimos anos em IE, tem sido aliada para auxiliar na melhoria de ambientes de aprendizagem [Borges et al. 2013]. Definida como o uso de elementos de jogos em ambientes não games [Deterding et al. 2011a], espera-se neste trabalho aliar IM's e gamificação, na intenção de apresentar novas contribuições para a IE. Para tal, pretende-se discutir a possível colaboração das mecânicas de jogos para a identificação, de forma automática, das inteligências que se destacam no aprendiz. Também pretende-se verificar se, com a gamificação em um ambiente adaptativo baseado em IM's, é possível encontrar características (tais como engajamento e maximização do aprendizado por parte do estudante), que já foram vistas em outros trabalhos [Borges et al. 2013].

Este artigo está organizado como se segue. A seção 2 apresenta a adaptatividade, mostrando os tipos, métodos utilizados para a criação e as abordagens usadas para a modelagem do estudante. A seção 3 apresenta a gamificação no contexto educacional, mostrando as principais mecânicas de jogos e as vantagens em utilizá-las, ao final são 
apresentados trabalhos brasileiros que utilizaram gamificação. A seção 4 descreve sobre o desafio na criação de um ambiente adaptativo baseado em inteligências múltiplas com uso de gamificação, apresentando as propostas trazidas até aqui. A seção 5 traz as considerações finais discutindo como o desafio proposto pode colaborar com a educação através da Informática.

\section{A Educação e a Adaptatividade}

Apesar da aprendizagem adaptativa conduzida por sistemas já apresentarem benefícios educacionais, ainda existem algumas lacunas a serem preenchidas para a melhor prática do ensino. Esta seção apresentará uma visão geral da adaptatividade no contexto educacional, associando os trabalhos já realizados junto com as teorias relacionadas à aprendizagem.

Quando se trata de adaptatividade no contexto da aprendizagem, existem três pilares a serem discutidos, segundo [Group et al. 2010], são eles:

- Individualização: São classificados neste item ambientes que esperam o mesmo objetivo de aprendizagem para diferentes estudantes, apresentando materiais distintos, adaptando ao perfil, respeitando o ritmo de cada aprendiz.

- Diferenciação: Neste caso, os objetivos de aprendizagem são os mesmos, porém os métodos ou a abordagem das instruções variam de acordo com a preferência do estudante.

- Personalização: Este item refere a ambientes que conseguem variar tanto os objetivos quanto os materiais que serão apresentados ao estudante de acordo com a classificação colocada a cada aprendiz.

Existem diferentes estratégias utilizadas por pesquisadores para a melhor individualização da aprendizagem, dentre elas as IM's. Segundo Gardner [Gardner 1983], o ser humano possui várias inteligências, sendo que, para determinadas áreas de atuação, algumas delas podem destacar enquanto outras não. De acordo com esta teoria, oito inteligências foram identificadas e são denominadas Linguística, musical, lógico-matemática, visual-espacial, corporal-cinestésica, interpessoal, intrapessoal e naturalista. Cogita-se ainda a possibilidade de uma nona inteligência (existencial), conforme Figura 1. Desta forma, para cada indivíduo, os níveis das IM's devem variar, tornando individual a forma de aprendizagem.

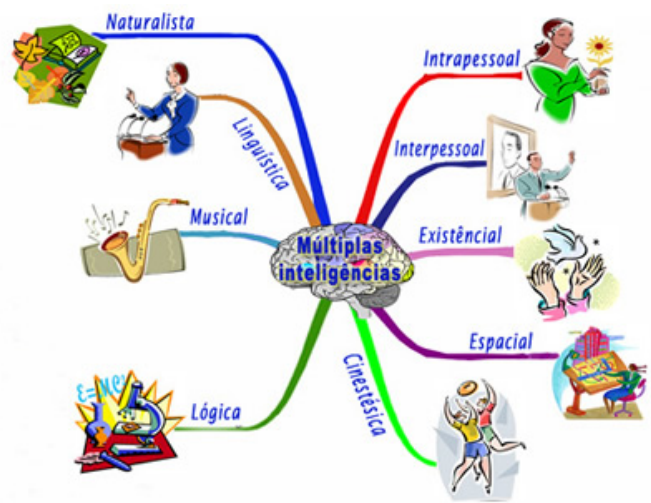

Figura 1. As Inteligências Múltiplas de Gardner [Barros 2015] 
Outra estratégia também utilizada na adaptatividade em ambientes de aprendizagem são os EA. Segundo pesquisas [Feldman et al. 2015, Valaski et al. 2011], existem várias recomendações de EA, porém, os dois estilos mais utilizados são os propostos por [Felder and Silverman 1988] e [Kolb and Kolb 2005]. As IM's, se comparadas aos EAs, referem-se ao que o indivíduo é possível realizar, às habilidades, enquanto os EAs referem-se às preferências no uso das habilidades [Kelly and Tangney 2006].

No Brasil, alguns pesquisadores têm esforçado para a detecção dos EA's e recomendação de Objetos de Aprendizagem, gerando ambientes adaptativos. [Silva and Dorça 2014] apresentam uma abordagem automática para personalização do processo de ensino baseada nos EA de Felder e Silverman. [Mühlbeier and Mozzaquatro 2012] faz uma classificação do EA do estudante através de um questionário, podendo assim realizar a individualização do processo de ensino / aprendizagem. [de Moura and Fernandes 2012] apresentam uma proposta de um modelo computacional para a escolha de OAs baseado nos EA de Kolb e em IM's.

Dentre as oito IM's, Barbosa [Barbosa et al. 2004] cita que quatro delas (Linguístico-Verbal (LV), Lógico-Matemática (LM), Visual-Espacial (VE) e CinestésicoCorporal (CC)) permitem associação com mídias tais como vídeos, apresentações e animações. Assim, para tais IM's é possível construir Objetos de Aprendizagem (OA) que forneçam conteúdos personalizados para o estudante. As três primeiras IM's (LV, LM e VE) também são apresentadas na construção do sistema adaptativo Educe [Kelly and Tangney 2006], que também apontam uma quarta, a Musical, pois acredita-se no alto poder emocional da música. Segundo Barbosa [Barbosa et al. 2004], o trabalho reduzido com IM's não traz prejuízos ao processo, pois as mesmas são independentes entre si.

Como já dito, IM's podem ser medidas através de questionários. Um questionário clássico para a medição possui 119 questões e provê um perfil descritivo das IM's do indivíduo [Feldman et al. 2015]. Outros instrumentos foram desenvolvidos tentando minimizar o número de questões e obter o mesmo resultado [Barbosa et al. 2004].

É possível notar que, na criação de ambientes adaptativos, há dois métodos de obtenção de informação para a modelagem do estudante. Segundo Graf [Graf et al. 2009], os métodos podem ser classificados como colaborativo e automático. Uma abordagem colaborativa permite que o aprendiz forneça informações de forma explicita, através de preenchimento de questionários, para a formação e atualização do seu modelo. Já na abordagem automática, através do comportamento e ações dos estudantes no sistema, o modelo é construído e atualizado de forma automática.

\section{Gamificação no contexto educacional}

Os sistemas dotados de inteligência desenvolvidos para o ensino procuram cada vez mais ferramentas que possam não somente enriquecer a forma como o conteúdo é apresentado, mas também fornecer um ambiente confortável para o estudante, levando estímulo e o tornando engajado no processo. Neste aspecto, a gamificação, definida como o uso de elementos de jogos em ambientes não games [Deterding et al. 2011b], vem sendo trabalhada nos últimos anos em ambientes educacionais.

Segundo Bunchball [Bunchball 2010], para a técnica de gamificação, algumas das mecânicas de jogos mais comuns são: pontos; níveis; desafios, troféus, emblemas / meda- 
lhas e conquistas; bens virtuais; placar de classificação, ranking e tabela de pontuação. A Tabela 1 apresenta alguns exemplos de mecânica de jogos e suas respectivas explicações. Ainda segundo Bunchball [Bunchball 2010], as mecânicas de jogos impulsionam as pessoas pelas dinâmicas.

Tabela 1. Algumas mecânicas de jogos [Bunchball 2010]

\begin{tabular}{|c|c|}
\hline \multicolumn{2}{|c|}{ Mecânicas de Jogos } \\
\hline Tipos & Significado \\
\hline Níveis & Indica que o usuário atingiu uma meta \\
\hline Desafios & $\begin{array}{c}\text { Representam missões para serem realizadas e } \\
\text { posteriormente geram recompensas }\end{array}$ \\
\hline $\begin{array}{c}\text { Troféus,Emblemas/ } \\
\text { Medalhas, Conquistas }\end{array}$ & $\begin{array}{c}\text { Reconhecimentos visíveis que o usuário conquistou } \\
\text { desafios ou atingiu níveis }\end{array}$ \\
\hline $\begin{array}{c}\text { Placar de Classificação, } \\
\text { Ranking, Tabela de pontuação }\end{array}$ & $\begin{array}{c}\text { Posiciona os usuários em relação aos demais } \\
\text { Recompensa }\end{array}$ \\
$\begin{array}{c}\text { É dada ao usuário após a ocorrência de uma ação } \\
\text { para motivá-lo a realizar aquilo novamente }\end{array}$ \\
\hline
\end{tabular}

Recompensa, status, realização, auto expressão, concorrência, altruísmo, etc., são os impulsionadores e gerados a partir de mecânicas de jogos. Por exemplo, a recompensa é dada ao usuário após a ocorrência de uma ação para motivá-lo a realizar aquela ação novamente. Em programas de fidelidade feitos por companhias aéreas, os pontos que são dados aos usuários através de parcerias e voos realizados, ao final são convertidos em passagens aéreas. Os usuários desta forma se sentem motivados em participar e se fidelizarem aos programas.

Em um ambiente educacional, o principal objetivo da gamificação tem sido a utilização de mecânicas de jogos para tornar o processo educativo mais interessante, iterativo e atrativo [Domínguez et al. 2013]. Borges [Borges et al. 2013], após realizar um mapeamento sistemático, verificou que, na educação, a gamificação tem atuado principalmente nas seguintes categorias: Aprimoramento de habilidades; Desafios; Guidelines; Engajamento; Maximização do aprendizado; Mudança de comportamento e socialização.

A gamificação, segundo alguns pesquisadores da área de educação, pode apresentar problemas. [Muntean 2011], por exemplo, comenta o risco de se ter um ambiente gamificado e o estudante ficar preso à necessidade de receber recompensas para executar alguma atividade. Neste caso, o desenvolvedor deve preocupar em controlar também o comportamento do estudante, para que a gamificação seja um acréscimo para a motivação e não um fator dependente.

No Brasil algumas pesquisas têm sido voltadas para o uso de gamificação em sistemas educacionais. [Ferreira et al. 2015] utiliza a gamificação como um módulo para enriquecer um ambiente educacional ubíquo e o tornar mais sociável. [da Rocha Seixas et al. 2014, da Rocha Seixas et al. 2016] apresenta a gamificação como estratégia de engajamento de estudantes do ensino fundamental. $\mathrm{O}$ trabalho também apresenta uma visão bem detalhada sobre o que pode ser tratado como engajamento. [Neto et al. 2015] faz uma análise do uso da gamificação em uma turma do primeiro ano do ensino médio e verifica uma melhora na aprendizagem. Nos anos anteriores do Desafie 
também foi possível ver algumas propostas com uso de gamificação em Sistemas Tutores Inteligentes [Andrade et al. 2013, Andrade et al. 2014].

\section{Proposta: Um caminho para Vencer o Desafio}

Através das pesquisas apresentadas anteriormente foi possível ver alguns cenários já existentes e algumas questões em aberto para a Informática na Educação. O ensino orientado por sistemas para, principalmente, auxiliar na EaD é um contexto bem discutido mas que ainda apresentam lacunas a serem preenchidas, como, por exemplo, a modelagem automática do estudante, a motivação e o engajamento para a diminuição de evasão, o controle de acesso aos sistemas, etc.

$\mathrm{Na}$ intenção de contribuir com a IE, este trabalho tem como proposta o uso da gamificação em um ambiente de aprendizagem adaptativa baseado em Inteligências Múltiplas. Como as IM's são independentes entre si, a principio pretende-se expor um cenário somente com as quatro inteligências que [Barbosa et al. 2004] associou a mídias.

O ambiente deve ser constituído de quatro cenários distintos, cada um formado de acordo com os OAs classificados para a respectiva IM. Por exemplo, para o cenário correspondente à inteligência Linguista-Verbal, vídeos, materiais para escrever e promoção do debate são itens que devem existir estimulando o aprendizado do estudante que possui um perfil verbal.

Por ter a intenção de criar uma abordagem automática, o aprendiz não fornecerá nenhuma informação inicial, sendo que a criação e atualização do modelo será realizada de acordo com a interação do usuário com o sistema. Neste caso, métodos probabilísticos devem ser utilizados para construir o modelo do estudante, isto deve ao fato de lidar com um desconhecido. Em Sistemas Tutores Inteligentes, o uso de Redes Bayesianas são bem aceitos como método probabilístico para modelar, por exemplo, o conhecimento do estudante [Vier et al. 2015].

O ambiente também deve ter a individualidade como critério de adaptatividade. Desta forma, os aprendizes terão o mesmo objetivo, porém o ritmo e os OAs fornecidos serão individuais. A gamificação fornecerá as informações necessárias para o estudante compreender o nível de aprendizagem em cada cenário. A princípio o estudante poderá escolher um cenário ou será selecionado aleatório, sem a necessidade de preenchimento de questionários, como já é de praxe em alguns ambientes adaptativos. Neste cenário o estudante terá materiais para aquisição de conhecimento e avaliações ao término de cada módulo para aferir a aprendizagem. A mudança de cenário poderá ocorrer sempre que o estudante achar viável. Agentes atuando junto às mecânicas de jogos ajudarão o mesmo a compreender em qual cenário está tendo mais fracasso ou sucesso.

Após um certo uso do sistema, já será possível formar um modelo, visualizar qual inteligência do estudante está sobrepondo e, desta forma, recompensa poderá ser dada para o mesmo manter no cenário que a aprendizagem apresenta mais sucesso. O modelo estará em constante atualização decorrente do tempo gasto, das interações e dos resultados nas avaliações.

A gamificação, neste caso, não ficará restrita somente ao auxilio na modelagem do estudante e recomendação do material de acordo com sua IM. Como já apresentado em outros ambientes de aprendizagem, não é esperado diferente em um sistema baseado em 
inteligências múltiplas: É esperado que as mecânicas de jogos também possam estimular o engajamento e maximização do aprendizado, aprimorando assim as habilidades dos estudantes. Não pretende-se utilizar rankings, tabela de pontos e placar de classificação. A pontuação será individual, pois não tem a intenção de criar concorrência no ambiente.

\section{Considerações Finais}

Como apresentado no início deste artigo, a EaD no Brasil ainda enfrenta grandes problemas de evasão e grande queixa quanto à metodologia e aos ambientes virtuais de aprendizagem. A proposta apresentada aqui espera ajudar na solução deste problema atual. Com a criação de um ambiente com IM's e gamificação, pode possibilitar a motivação do estudante durante os estudos. $\mathrm{O}$ aprendiz poderá aprender com os materiais que mais possam despertar interesse e, através das mecânicas de jogo, se sentir engajado no processo educacional.

A individualidade na aprendizagem faz com que se crie um desafio durante a construção de sistemas educacionais. Com as propostas expostas anteriormente, espera-se um sistema de aprendizagem adaptativo, baseado em inteligências múltiplas, com uso de gamificação, para classificar estudantes de acordo com sua inteligência e também mantêlos motivados com uso de mecânicas de jogos.

Resumindo, os principais desafios discutidos são:

1. Determinar um modelo de estudante que seja capaz de obter informações relevantes sem o uso de questionários, utilizando a teoria de Inteligências Múltiplas;

2. Implementar atividades a serem realizadas através do computador que permitam manter a motivação do estudante, aumentar o aproveitamento no processo de ensino/aprendizagem e, principalmente, fornecer as informações que melhor identifiquem o perfil do estudante. Para tanto foi sugerida a gamificação;

3. Criar métodos probabilísticos para assistir a evolução do estudante; e

4. atualizar estas informações durante o processo a fim de acompanhar a evolução do estudante ou, até mesmo constatar que esta não ocorreu.

Trabalhos futuros tangem na integração do sistema construído a um Ambiente Virtual de Aprendizagem, de preferência o Moodle ${ }^{1}$, por ser mais utilizado em situações reais de ensino. Desta forma, permitirá maior número de acessos pelos estudantes. Também pretende-se criar módulos para tornar o ambiente colaborativo, talvez aproveitando as mecânicas de jogos, pois acredita-se que a colaboração possa enriquecer a aprendizagem e permitir a observação mais efetiva do comportamento do estudante.

\section{Referências}

Andrade, F., Chalco, G., and Isotani, S. (2014). Em direção à gamificação de sistemas tutores inteligentes: Aplicando a teoria de fluxo Ótimo no design instrucional de cenários de aprendizagem colaborativa. In Anais do DesafIE-IV Workshop de Desafios da Computação Aplicada à Educação, volume 1.

Andrade, F., Pedro, L., Lopes, A., Bittencourt, I., and Isotani, S. (2013). Desafio do uso de gamificação em sistemas tutores inteligentes baseados em web semântica. In Anais do DesafIE-III Workshop de Desafios da Computação Aplicada à Educação, volume 1.

\footnotetext{
${ }^{1}$ Moodle: disponível em http://moodle.com/
} 
Barbosa, A. T. R. et al. (2004). Mecanismo de adaptação baseado em redes neurais artificiais para sistemas hipermídia adaptativos. $\mathrm{PhD}$ thesis, Universidade Federal de Santa Catarina, Centro Tecnológico. Programa de Pós-graduação em Engenharia Elérica.

Barros, J. (2015). InteligÊncias mÚltiplas - novo conceito em educaÇÃo.

Borges, S. d. S., Reis, H. M., Durelli, V. H., Bittencourt, I. I., Jaques, P. A., and Isotani, S. (2013). Gamificação aplicada à educação: Um mapeamento sistemático. Simpósio Brasileiro de Informática na Educação, pages 234-243.

Bunchball, I. (2010). Gamification 101: An introduction to the use of game dynamics to influence behavior [white paper].

da Rocha Seixas, L., Gomes, A. S., and de Melo Filho, I. J. (2016). Effectiveness of gamification in the engagement of students. Computers in Human Behavior, 58:4863.

da Rocha Seixas, L., Gomes, A. S., Melo Filho, I. J., and Rodrigues, R. L. (2014). Gamificação como estratégia no engajamento de estudantes do ensino fundamental. In Anais do Simpósio Brasileiro de Informática na Educação, volume 25, page 559.

de Moura, F. F. and Fernandes, M. A. (2012). Uma proposta de um modelo computacional que usa pso para a escolha de objetos de aprendizagem baseado na espiral de kolb e nas inteligências múltiplas. In Anais do Simpósio Brasileiro de Informática na Educação, volume 23.

Deterding, S., Dixon, D., Khaled, R., and Nacke, L. (2011a). From game design elements to gamefulness: defining gamification. In Proceedings of the 15th international academic MindTrek conference: Envisioning future media environments, pages 9-15. ACM.

Deterding, S., Khaled, R., Nacke, L. E., and Dixon, D. (2011b). Gamification: Toward a definition. In CHI 2011 Gamification Workshop Proceedings, pages 12-15.

Domínguez, A., Saenz-de Navarrete, J., De-Marcos, L., Fernández-Sanz, L., Pagés, C., and Martínez-Herráiz, J.-J. (2013). Gamifying learning experiences: Practical implications and outcomes. Computers \& Education, 63:380-392.

Felder, R. M. and Silverman, L. K. (1988). Learning and teaching styles in engineering education. Engineering education, 78(7):674-681.

Feldman, J., Monteserin, A., and Amandi, A. (2015). Automatic detection of learning styles: state of the art. Artificial Intelligence Review, 44(2):157-186.

Fernandes, J., da Silva Ferreira, A., de Oliveira Nascimento, D. C., Shimoda, E., and Teixeira, G. F. (2014). Identificação de fatores que influenciam na evasão em um curso superior de ensino a distância. PerspectivasOnLine 2007-2010, 4(16).

Ferreira, H., Araújo, R. D., Souza, P., Chagas, S., Dorça, F., and Cattelan, R. (2015). Gamificação em ambientes educacionais ubíquos. In Anais do Simpósio Brasileiro de Informática na Educação, volume 26, page 509.

Gardner, H. (1983). Frames of mind: The theory of multiple intelligences. NY: Basics. 
Graf, S. et al. (2009). Advanced adaptivity in learning management systems by considering learning styles. In Proceedings of the 2009 IEEE/WIC/ACM International Joint Conference on Web Intelligence and Intelligent Agent Technology-Volume 03, pages 235-238. IEEE Computer Society.

Graf, S., Ives, C., et al. (2010). A flexible mechanism for providing adaptivity based on learning styles in learning management systems. In Advanced Learning Technologies (ICALT), 2010 IEEE 10th International Conference on, pages 30-34. IEEE.

Group, N. E. T. P. T. W. et al. (2010). Transforming american education: Learning powered by technology.

Kelly, D. and Tangney, B. (2006). Adapting to intelligence profile in an adaptive educational system. Interacting with Computers, 18(3):385-409.

Kolb, A. Y. and Kolb, D. A. (2005). Learning styles and learning spaces: Enhancing experiential learning in higher education. Academy of management learning \& education, 4(2):193-212.

Martins, C. Z., de Lima Terçariol, A. A., and Gebran, R. A. (2016). Percepções do tutor: a evasão de alunos de curso de administração na modalidade a distância. REVISTA ELETRÔNICA PESQUISEDUCA, 7(14):470-492.

Mühlbeier, A. R. and Mozzaquatro, P. M. (2012). Estilos e estratégias de aprendizagem personalizadas a alunos das modalidades presenciais e a distância. Revista Brasileira de Informática na Educação, 20:132.

Muntean, C. I. (2011). Raising engagement in e-learning through gamification. In Proc. 6th International Conference on Virtual Learning ICVL, pages 323-329.

Neto, A., da Silva, A. P., and Bittencourt, I. I. (2015). Uma análise do impacto da utilização de técnicas de gamificação como estratégia didática no aprendizado dos alunos. In Anais do Simpósio Brasileiro de Informática na Educação, volume 26, page 667.

Silva, D. H. and Dorça, F. A. (2014). Uma abordagem automática para personalização do processo de ensino baseada em estilos de aprendizagem em sistemas adaptativos e inteligentes para educação a distância. Revista Brasileira de Informática na Educação, 22(02):1.

Valaski, J., Malucelli, A., and Reinehr, S. (2011). Revisão dos modelos de estilos de aprendizagem aplicados à adaptação e personalização dos materiais de aprendizagem. In Anais do Simpósio Brasileiro de Informática na Educação, volume 1.

Vier, J., Gluz, J., and Jaques, P. A. (2015). Empregando redes bayesianas para modelar automaticamente o conhecimento dos alunos em lógica de programação. Revista Brasileira de Informática na Educação, 23(02):45. 